Test kits were returned individually and results issued to the health care staff who disseminated them to individual prisoners. Should a prisoner have a positive test result, arrangements will be made with prison healthcare staff for telephone assessment to be undertaken by a Specialist Screening Practitioner (SSP). Prior arrangement with the prison will ensure that the prisoner is brought to the medical centre to undergo this assessment.

Consideration has to be given to posting of bowel preparation medication and for prisoners who needed to undergo colonoscopy. Collaboration with prison staff was needed to ensure prisoners were given equal opportunity to participate in the programme without breaching prison security policies.

Results The pilot was established in two prisons and is currently being rolled out in another. Uptake has been encouraging and a complete data set for the pilot phase will be presented in June. To date all prisoners who participated have received negative results and the positive pathway has not yet been tested.

Challenges encountered included engagement of the healthcare teams within prisons due to staffing levels and varying viewpoints towards health care and the concept of informed choice and consent. Conclusion By engaging and developing this service within Welsh prisons, BSW has extended its population based screening programme to a vulnerable group and are committed to formal evaluation and service improvement where necessary.

Disclosure of Interest None Declared.

\section{PTH-145 INVESTIGATION OF PARTICIPANT RESPONSES AND IMPLICATIONS FOR UPTAKE FOLLOWING SPOILT TEST KITS IN THE WELSH BOWEL CANCER SCREENING PROGRAMME}

doi:10.1136/gutjnl-2013-304907.632

${ }^{1} \mathrm{H}$ Beer, ${ }^{1, *} \mathrm{H}$ Heard. ${ }^{1}$ Screening Division, Public Health UK, Cardiff, UK

Introduction Participants return kits to the Bowel Screening Wales (BSW) laboratory for testing. It is not possible to test some kits and these are spoilt. The BSW Laboratory has seen an increase in spoilt rate from 1.8\% in Sep 2011 to 3.2\% in Sep 2012, most related to a change in laboratory procedure to reject samples because identifiers written on the test kit did not match details held on the BSW Information Management System.

BSW analysed the effect this change in policy may have had on response rates longer term within the programme in the context of a decreasing uptake rate (currently at $53 \%$ ). This was based on the hypothesis that not all participants might complete a second test kit. Methods Participants were recorded as final non responders if no test kit has been received within six months of the initial invitation. Participants with a spoilt result validated from Oct 2008 to May 2012 were included in the analysis.

Results During the time period 8400 test kits were spoilt by the laboratory, $78 \%$ returned the test kit that was sent with the spoilt result and $80 \%$ returned another test kit sent in the same invitation episode. $80 \%$ returned FOB (Faecal Occult Blood test) kits and 90\% FIT (Faecal Immunochemical Test) kits sent following an equivocal FOB result. If participants did respond, it was usually received within 4 weeks $(70 \%)$, with another $6 \%$ returning their kit within 4-8 weeks later and $4 \%$ sending in their kit more than two months later.

The helpline received numerous calls from participants who were disappointed to have had their kit spoilt because of identity reasons. This may have potentially resulted in a decrease in motivation on part of the participant.

For those participants who did not send another kit back during the same episode (20\%), to date only half have been re-invited as part of their next routine recall allowing six months follow up. Of these participants re-invited in a new episode $74 \%$ have not responded, (74\% for FOB kits and $67 \%$ for FIT kits).

Figures for spoilt FOB were similar for males and females, with younger participants less like to respond. Figures for FIT kits show men more likely to return their later kit (this may need to be interpreted with caution in view of the limited sample size in this group). Conclusion $20 \%$ of participants who respond and have a spoilt test result do not respond to another kit issued in the same invitation episode. These participants appear less likely to attend screening during the next invitation episode, $26 \%$ responded (allowing six months follow up). With 5000 spoilt test results issued per annum, 260 participants may not take part in the programme again. We suggest that further studies may help towards directing efforts for increased uptake in this group.

Disclosure of Interest None Declared.

\section{PTH-146 OPTIMISING THE SCREENING STRATEGY TO REDUCE INTERVAL CANCERS - INITIAL EXPERIENCE IN THE WELSH BOWEL SCREENING PROGRAMIME}

doi:10.1136/gutjinl-2013-304907.633

${ }^{1} \mathrm{H}$ Beer, ${ }^{1, " H}$ Heard. 'Screening Division, Public Health UK, Cardiff, UK

Introduction Analysis of interval bowel cancer rates within two years of a negative screening test kit result demonstrates rates twice as high following an equivocal FOB (Faecal Occult Blood test) with a negative FIT (Faecal Immunochemical Test), compared to a negative FOB. This analysis looks at the time to bowel cancer diagnosis following a negative FIT result to see whether participants may benefit from being recalled earlier for bowel screening than the current two year strategy.

Methods In Wales, the routine recall interval is two years. A cohort of participants with negative results validated between Oct 2008 and August 2010 were reviewed, with two years follow up data. These records were compared with all screen detected and symptomatic bowel cancers diagnosed in the two year follow up time period. The time interval from the negative screening result to their diagnosis of bowel cancer was recorded.

Results In the analysis time period, 9000 participants were issued with a negative FIT screening test result, 22 went on to develop bowel cancer within the next 2 years $(0.24 \%)$. This compared to $0.14 \%$ of participants who had a negative $\mathrm{FOB}$ and were returned to routine recall (almost significantly different).

Of the 22 participants with cancer diagnosed following a negative FIT screening test result, the mean time to diagnosis was 366 days (median 316 days). However this varied depending on gender and five year age group.

$29 \%$ women had a bowel cancer diagnosed within 3 months of their negative test result, compared to $7 \%$ men. At 6 months $20 \%$ men had been diagnosed with cancer. Similarly with the younger age group, $18 \%$ of participants aged $60-64$ years had a bowel cancer diagnosed within 3 months, compared to $9 \%$ of those aged 65 years and older.

Analysing the potential costs of a tailored approach towards these equivocal test results, the cost to the bowel screening programme in Wales per year would be around 5,000 invitation letters with test kits to be posted out earlier (rather than at the two year routine recall interval). These potentially have the ability to diagnose the expected 10 cancers that would occur in the following twelve months.

Conclusion Women and the younger age group (60-64 years) may benefit from an early repeat test either at three months or immediately following their negative FIT test result. Men and the older age group ( 65 years and above) may benefit from an early repeat test at six months. It is unclear whether these are false negatives or true interval cancers but these preliminary results would suggest that if validated in a larger cohort, a tailored approach to equivocal FOB tests may optimise yield and potentially reduce the incidence of interval cancers.

Disclosure of Interest None Declared. 